\title{
On Interval Edge Colorings of Biregular Bipartite Graphs With Small Vertex Degrees
}

Carl Johan Casselgren and Bjarne Toft

\author{
Linköping University Post Print
}

\section{Tweet}

N.B.: When citing this work, cite the original article.

Original Publication:

Carl Johan Casselgren and Bjarne Toft, On Interval Edge Colorings of Biregular Bipartite Graphs With Small Vertex Degrees, 2015, Journal of Graph Theory, (80), 2, 83-97.

http://dx.doi.org/10.1002/jgt.21841

Copyright: Wiley: 12 months

http://eu.wiley.com/WileyCDA/

Postprint available at: Linköping University Electronic Press

http://urn.kb.se/resolve?urn=urn:nbn:se:liu:diva-121093 


\title{
On interval edge colorings of biregular bipartite graphs with small vertex degrees
}

\author{
Carl Johan Casselgren* \\ Department of Mathematics \\ Linköping University \\ SE-581 83 Linköping, Sweden
}

\author{
Bjarne Toft ${ }^{\dagger}$ \\ Department of Mathematics \\ University of Southern Denmark \\ DK-5230 Odense, Denmark
}

August 29, 2014

\begin{abstract}
A proper edge coloring of a graph with colors $1,2,3, \ldots$ is called an interval coloring if the colors on the edges incident to each vertex form an interval of integers. A bipartite graph is $(a, b)$-biregular if every vertex in one part has degree $a$ and every vertex in the other part has degree $b$. It has been conjectured that all such graphs have interval colorings. We prove that all $(3,6)$-biregular graphs have interval colorings and that all $(3,9)$ biregular graphs having a cubic subgraph covering all vertices of degree 9 admit interval colorings. Moreover, we prove that slightly weaker versions of the conjecture hold for $(3,5)$ biregular, (4,6)-biregular and (4,8)-biregular graphs. All our proofs are constructive and yield polynomial time algorithms for constructing the required colorings.
\end{abstract}

\section{Introduction}

A proper edge coloring of a graph $G$ with colors $1,2,3, \ldots$ is called an interval coloring (or consecutive coloring) if the colors on the edges incident to each vertex form an interval of integers. The notion of interval colorings was introduced by Asratian and Kamalian [4] (available in English as [3]), motivated by the problem of finding compact school timetables, that is, timetables such that the lectures of each teacher and each class are scheduled in consecutive periods. Hansen [11] described another scenario (obtained from Jesper BangJensen): a school wishes to schedule parent-teacher conferences in time slots so that every person's conferences occur in consecutive slots. A solution exists if and only if the bipartite graph with vertices for parents and teachers and edges for the required meetings has an interval coloring.

All regular bipartite graphs have interval colorings, since they decompose into perfect matchings. Not every (non-bipartite) graph has an interval coloring, since a graph $G$ with

${ }^{*}$ E-mail address: carl.johan.casselgren@liu.se Work done while the author was a postdoc at University of Southern Denmark. Research supported by SVeFUM.

${ }^{\dagger} E$-mail address: btoft@imada.sdu.dk 
an interval coloring must have a proper $\Delta(G)$-edge-coloring [4] (obtained from the interval coloring by taking colors modulo $\Delta(G)$ ). Sevastjanov [20] proved that determining whether a bipartite graph has an interval coloring is $\mathcal{N} \mathcal{P}$-complete, and he also gave the first concrete example of a bipartite graph having no interval coloring. Nevertheless, trees [11, 3], complete bipartite graphs [11,4], grids [10], and outerplanar bipartite graphs $[9,5]$ all have interval colorings. Hansen [11] showed that all bipartite graphs of maximum degree 3 have interval 4-colorings, and Giaro [8] showed that one can decide in polynomial time whether bipartite graphs with maximum degree 4 have interval 4-colorings.

A bipartite graph with parts $X$ and $Y$ is called $(a, b)$-biregular if all vertices of $X$ have degree $a$ and all vertices of $Y$ have degree $b$. In this paper we study the following well-known conjecture $[11,14,21]$ :

Conjecture 1.1. Every $(a, b)$-biregular graph has an interval coloring.

By results of [11] and [13], all (2,b)-biregular graphs admit interval colorings (the result for odd $b$ was obtained independently by Kostochka [15]). Several sufficient conditions for a $(3,4)$-biregular graph to admit an interval coloring has been obtained $[2,7,19,22]$. In [6] we give a sufficient condition for a $(3,5)$-biregular graph to admit an interval coloring. However, in general, Conjecture 1.1 is still wide open; the smallest unsolved case is $(a, b)=(3,4)$.

In the paper we present a simple proof of the following.

Theorem 1.2. Every (3,6)-biregular graph has an interval 7-coloring.

Note that the number of colors used in Theorem 1.2 is best possible in the sense that the problem to determine whether a $(3,6)$-biregular graph has an interval 6 -coloring is $\mathcal{N} \mathcal{P}$ complete [1]. Using Theorem 1.2, we present a family of (3,9)-biregular graphs having interval colorings: such a graph has an interval 10-coloring if it has a 3-regular subgraph covering all vertices of degree 9 .

In this paper we also consider two natural generalizations of interval colorings. For a set $A$ of positive integers, $A$ is near-interval if there is a positive integer $n(A)$ such that $A \cup\{n(A)\}$ is an interval; $A$ is cyclic interval modulo $t$ if either $A$ or $\{1, \ldots, t\} \backslash A$ is an interval. A proper $t$-edge coloring $f$ of a graph $G$ is a near-interval coloring if the set of colors on the edges incident to any vertex of $G$ is near-interval; $f$ is a cyclic interval coloring if the set of colors on the edges incident to any vertex is cyclic interval modulo $t$.

Near-interval colorings were first studied in [18] under the name interval $(t, 1)$-colorings. Therein the authors e.g. give examples of bipartite graphs having no near-interval colorings, and they provide bounds on the number of colors in near-interval colorings for different families of graphs. Cyclic interval colorings are studied in e.g. [17, 16]. In particular, the general question of determining whether a bipartite graph $G$ has a cyclic interval coloring is $\mathcal{N} \mathcal{P}$-complete [16] and there are concrete examples of connected bipartite graphs having no cyclic interval coloring [17]. Trivially, any bipartite graph with an interval coloring also has a cyclic interval coloring with $\Delta(G)$ colors, but the converse does not hold [17].

In [6] we give some conditions for a $(3,5)$-biregular graph to admit a cyclic interval coloring. Here, we establish that slightly weaker versions of Conjecture 1.1 hold for $(3,5)$ biregular, (4,6)-biregular and (4,8)-biregular graphs by proving the following:

(i) Every $(3,5)$-biregular graph has a near-interval coloring; 
(ii) Every (4,6)-biregular graph has a near-interval coloring;

(iii) Every (4,8)-biregular graph has a cyclic interval coloring.

In Section 2 we give the proof of Theorem 1.2. Statements (i) and (ii) are proved in Section 3 , where we also present an infinite family of $(4,6)$-biregular graphs having interval colorings. In Section 4 we consider (4,8)-biregular graphs: we prove (iii) and briefly discuss the problem of constructing interval colorings of $(4,8)$-biregular graphs. The proofs of these results are all constructive and yield polynomial algorithms for constructing the corresponding colorings.

\section{Proof of the main result}

In this section we prove Theorem 1.2. First we introduce some notation and also state some preliminary results. Throughout the paper, we use the notation $G=(X, Y ; E)$ for a bipartite graph $G$ with bipartition $(X, Y)$ and edge set $E=E(G)$. We use the convention that if $G=(X, Y ; E)$ is $(a, b)$-biregular, then the vertices in $X$ have degree $a$. We denote by $d_{G}(v)$ the degree of a vertex $v$ in $G$, and by $N_{G}(v)$ the set of vertices adjacent to $v$ in $G$. If $V^{\prime} \subseteq V(G)$, then $N_{G}\left(V^{\prime}\right)=\cup_{v \in V^{\prime}} N_{G}(v)$, and $d_{G}\left(V^{\prime}\right)=\sum_{v \in V^{\prime}} d_{G}(v)$. The maximum degree in $G$ is denoted by $\Delta(G)$, and the minimum degree in $G$ is denoted by $\delta(G)$.

For an edge coloring $\varphi$ of a graph $G$, let $M(\varphi, i)=\{e \in E(G): \varphi(e)=i\}$. If $e \in M(\varphi, i)$, then $e$ is colored $i$ under $\varphi$. For a vertex $v \in V(G)$, we say that a color $i$ appears at $v$ under $\varphi$ if there is an edge $e$ incident to $v$ with $\varphi(e)=i$, and we set

$$
\varphi(v)=\{\varphi(e): e \in E(G) \text { and } e \text { is incident to } v\} .
$$

If $c \notin \varphi(v)$, then $c$ is missing at $v$ under $\varphi$. Moreover, if $\varphi(v)=\{c\}$, that is, $\varphi(v)$ is singleton, then $\varphi(v)$ usually denotes the color $c$ rather than the set $\{c\}$. In all the above definitions, we often leave out the explicit reference to a coloring $\varphi$, if the coloring is clear from the context.

We shall say that a proper edge coloring of a graph $G$ using positive integers is interval at a vertex $v \in V(G)$ if the colors on the edges incident to $v$ form an interval of integers. For the proof of Theorem 1.2, we will use the following result (which easily follows from Petersen's 2 -factor theorem for regular graphs of even degree).

Theorem 2.1. [11] If $G$ is (2,2k)-biregular for some positive integer $k$, then $G$ has an interval $2 k$-coloring where for each vertex $x$ of degree 2 , there is a positive integer $j$ such that the edges incident with $x$ are colored $2 j-1$ and $2 j$ (i.e. the smaller color is always odd).

Proof of Theorem 1.2. Let $G=(X, Y ; E)$ be a $(3,6)$-biregular graph. Define a new graph $H$ from $G$ by replacing each vertex $y$ of degree 6 in $G$ by two vertices $y^{\prime}$ and $y^{\prime \prime}$ of degree 3 , where $y^{\prime}$ is adjacent to three of the neighbors of $y$ and $y^{\prime \prime}$ is adjacent to the other three neighbors of $y$. The graph $H$ is 3 -regular, so by Hall's condition it has a perfect matching $M$. In $G, M$ induces a subgraph $F$ in which each vertex in $Y$ has degree 2 and each vertex in $X$ has degree 1.

The graph $G-E(F)$ is $(2,4)$-biregular, so by Theorem 2.1, there is an interval 4-coloring $f$ of $G-E(F)$ such that each vertex of $X$ receives colors 1 and 2 or 3 and 4 on its incident edges. We define a new proper edge coloring $f^{\prime}$ from $f$ by replacing colors 1 and 2 , with colors 
2 and 3, respectively; and colors 3 and 4 by colors 5 and 6 , respectively. Thus $f^{\prime}$ is a proper edge coloring of $G-E(F)$ using colors $2,3,5,6$ and for each vertex $x \in X, f^{\prime}(x)=\{2,3\}$ or $f^{\prime}(x)=\{5,6\}$.

We will now define an edge coloring $g$ of $F$ so that $g$ and $f^{\prime}$ together form an interval 7-coloring of $G$. For each vertex $y \in Y$, we color the edges of $F$ incident to $y$ according to the following. Let $x$ and $x^{\prime}$ be the neighbors of $y$ in $F$.

- If $f^{\prime}(x)=f^{\prime}\left(x^{\prime}\right)=\{2,3\}$, then we set $g(x y)=1$ and $g\left(x^{\prime} y\right)=4$;

- if $f^{\prime}(x)=f^{\prime}\left(x^{\prime}\right)=\{5,6\}$, then we set $g(x y)=4$ and $g\left(x^{\prime} y\right)=7$;

- if $f^{\prime}(x)=\{2,3\}$ and $f^{\prime}\left(x^{\prime}\right)=\{5,6\}$, then we set $g(x y)=4$ and $g\left(x^{\prime} y\right)=7$.

The colorings $f^{\prime}$ and $g$ together form an interval 7-coloring of $G$.

Remark 1. Since Theorem 2.1 is true also in the multigraph setting (see e.g. [11]), in particular, every $(2,4)$-biregular multigraph has an interval 4-coloring such that each vertex of degree 2 receives colors 1,2 or 3,4 on its incident edges, the proof of Theorem 1.2 is also valid in the multigraph setting. Thus, in fact every $(3,6)$-biregular multigraph has an interval 7-coloring.

Remark 2. To see that not every $(3,6)$-biregular graph has an interval 6-coloring, take the graph $G$ to consist of two disjoint copies of the complete graph $K_{7}$ on 7 vertices. Add seven new vertices to $G$ and extend each of the 42 edges of $G$ to a 3 -set by adding one of the new vertices such that each of the seven new vertices is in exactly six 3 -sets. The result is a 6-regular 3-uniform hypergraph $H$. Consider the corresponding $(3,6)$-biregular graph with partite sets $E(H)$ and $V(H)$ and an edge joining $e \in E(H)$ to $x \in V(H)$ whenever $e$ contains $x$. An interval 6-coloring of this graph corresponds to a factorization of $H$ into two 3 -factors. In turn a 3-factor of $H$ corresponds to a 3-factor in $G$ and hence in $K_{7}$. But no 3-regular graph has seven vertices.

Using Theorem 1.2, we can prove that a particular family of (3,9)-biregular graphs admit interval colorings.

Corollary 2.2. If $G$ is a $(3,9)$-biregular graph having a 3-regular subgraph covering all vertices of degree 9 , then $G$ has an interval 10-coloring.

Proof. Let $G=(X, Y ; E)$ be a $(3,9)$-biregular graph having a 3-regular subgraph $F$ covering all vertices of degree 9 in $G$. Let $X^{\prime}$ be the set of vertices of $F$ that have degree 3 in $G$. Then $G-X^{\prime}$ is $(3,6)$-biregular, and so it has an interval 7-coloring $f$. Suppose that $f$ uses colors $1, \ldots, 7$. Then for each vertex $y \in Y$, we have $f(y)=\{1, \ldots, 6\}$ or $f(y)=\{2, \ldots, 7\}$.

We will now define a proper edge coloring of $F$. By Hall's condition, $F$ has a perfect matching $M$. We define the proper edge coloring $g$ of $F$ in the following way. Let $e \in M$.

- If $e$ is incident with a vertex $y \in Y$ such that $f(y)=\{2, \ldots, 7\}$, then we color $e$ with 10 ;

- if $e$ is incident with a vertex $y \in Y$ such that $f(y)=\{1, \ldots, 6\}$, then we color $e$ with 7 . 


$x_{1} \bullet \quad x_{2} \bullet \quad x_{3} \bullet \quad x_{4} \bullet \quad \cdots \cdot \ldots$

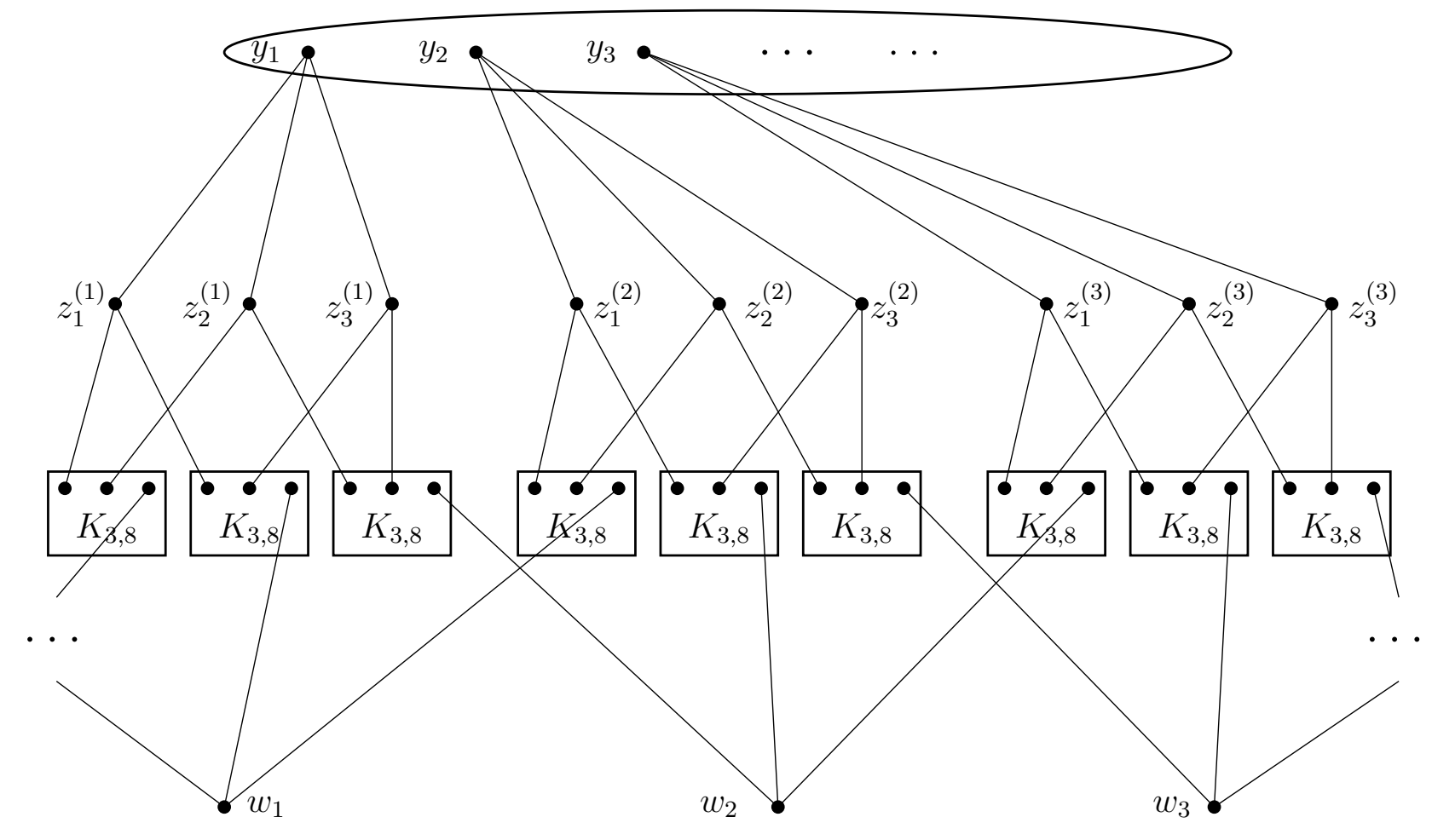

Figure 1: The graph $H=H(G)$.

This yields a coloring of the edges of $M . F-M$ is 2-regular so by König's edge coloring theorem, we may define $g$ on $F-M$ by letting it be a proper edge coloring using colors 8 and 9. The edge colorings $f$ and $g$ form an interval 10-coloring of $G$.

Next, we prove that the number of colors used in Corollary 2.2 is best possible. Let $G=(X, Y ; E)$ be a $(3,9)$-biregular graph. If $G$ has a decomposition into three edge-disjoint 3-regular subgraphs $G_{1}, G_{2}, G_{3}$, then we may construct an interval 9-coloring of $G$ by properly coloring the edges of $G_{i}$ with colors $3 i-2,3 i-1,3 i$, for $i=1,2,3$ (using König's edge coloring theorem). Conversely, if $G$ has an interval 9-coloring using colors $1, \ldots, 9$, then the subgraph $G_{i}$ induced by edges colored $3 i-2,3 i-1,3 i$ is 3 -regular and covers $Y$, for $i=1,2,3$. Hence, we have the following:

Proposition 2.3. A (3,9)-biregular graph $G$ has an interval 9-coloring if and only if it admits a decomposition into three edge-disjoint 3-regular subgraphs.

Now, let $G=(X, Y ; E)$ be a $(3,6)$-biregular graph. We shall construct a $(3,9)$-biregular graph $H=H(G)$ from $G$. Since $d_{G}(X)=d_{G}(Y)$, we have that $|X|=2 k$ and $|Y|=k$ for 
some positive integer $k$. Suppose that $X=\left\{x_{1}, \ldots, x_{2 k}\right\}$ and $Y=\left\{y_{1}, \ldots, y_{k}\right\}$, and let

$$
Z=\left\{z_{1}^{(1)}, z_{2}^{(1)}, z_{3}^{(1)}, z_{1}^{(2)}, z_{2}^{(2)}, z_{3}^{(2)}, \ldots, z_{1}^{(k)}, z_{2}^{(k)}, z_{3}^{(k)}\right\} \text { and } W=\left\{w_{1}, \ldots, w_{k}\right\}
$$

be two sets of $3 k$ and $k$ new vertices, respectively. Moreover, let $L$ be a new graph consisting of three pairwise disjoint copies $K_{3,8}^{(1)}, K_{3,8}^{(2)}, K_{3,8}^{(3)}$ of the complete bipartite graph $K_{3,8}$, where the vertices in $K_{3,8}^{(i)}$ of degree 8 are denoted by $a_{i 1}, a_{i 2}, a_{i 3}$, respectively, $i=1,2,3$. We take $k$ pairwise disjoint copies $L_{1}, \ldots, L_{k}$ of the graph $L$, and denote the vertices in $L_{j}$ corresponding to $a_{11}, \ldots, a_{33}$, by $a_{11}^{(j)}, \ldots, a_{33}^{(j)}, j=1, \ldots, k$. Set $\mathcal{L}=\cup_{j=1}^{k} L_{j}$.

For each $j=1, \ldots, k$, we define a set $E_{j}$ of edges by letting

$$
\begin{aligned}
E_{j}= & \left\{y_{j} z_{1}^{(j)}, y_{j} z_{2}^{(j)}, y_{j} z_{3}^{(j)}\right\} \cup\left\{w_{j} a_{33}^{(j-1)}, w_{j} a_{23}^{(j)}, w_{j} a_{13}^{(j+1)}\right\} \cup \\
& \left\{z_{1}^{(j)} a_{11}^{(j)}, z_{1}^{(j)} a_{21}^{(j)}, z_{2}^{(j)} a_{12}^{(j)}, z_{2}^{(j)} a_{31}^{(j)}, z_{3}^{(j)} a_{22}^{(j)}, z_{3}^{(j)} a_{32}^{(j)}\right\},
\end{aligned}
$$

where all indices are taken modulo $k$. We now define the graph $H=H(G)$ by setting

$$
V(H)=V(G) \cup V(\mathcal{L}) \cup W \cup Z \text { and } E(H)=E(G) \cup E(\mathcal{L}) \cup E_{1} \cup \cdots \cup E_{k} .
$$

The graph $H$ is $(3,9)$-biregular and contains $G$ as a subgraph (see Figure 1 ). Moreover, it is straightforward to verify that $H$ has a decomposition into 3-regular subgraphs if and only if $G$ has a 3-regular subgraph covering $Y$.

Suppose that $G$ has no 3-regular graph covering $Y$. (Such a $(3,6)$-biregular graph is easy to construct, an explicit example appears in Remark 2.) Then the graph $H=H(G)$ has a 3 -regular subgraph covering all vertices of degree 9 , but no decomposition into edge-disjoint 3-regular subgraphs; that is, $H$ satisfies the hypothesis of Corollary 2.2 and by Proposition 2.3 it has no interval 9-coloring. Hence, the number of colors used in Corollary 2.2 is best possible.

Furthermore, in [1] the following was proved:

Proposition 2.4. [1] It is an $\mathcal{N} \mathcal{P}$-complete problem to determine if a $(3,6)$-biregular graph has a 3-regular subgraph covering all vertices of degree 6.

Remark 3. It is not hard to verify that a $(3,6)$-biregular graph has an interval 6-coloring if and only if it has a 3-regular subgraph covering all vertices of degree 6; so Proposition 2.4 is the result underlying the fact that the problem to determine if a $(3,6)$-biregular graph has an interval 6-coloring is $\mathcal{N} \mathcal{P}$-complete [1].

Now, let $G=(X, Y ; E)$ be a $(3,6)$-biregular graph. Since the graph $H=H(G)$ constructed above admits a decomposition into 3-regular subgraphs if and only if $G$ has a 3regular subgraph covering $Y$, it follows from Proposition 2.3 and 2.4 that the problem to determine if a $(3,9)$-biregular graph has an interval 9-coloring is $\mathcal{N} \mathcal{P}$-complete. In fact, the problem of deciding whether a $(3,9)$-biregular graph satisfies the hypothesis of Corollary 2.2 is also $\mathcal{N} \mathcal{P}$-complete.

Proposition 2.5. The problem of determining whether a $(3,9)$-biregular graph has a 3-regular subgraph covering all vertices of degree 9 is $\mathcal{N} \mathcal{P}$-complete.

The proof of Proposition 2.5 is deferred to the appendix. 


\section{$3 \quad$ Near-interval colorings}

In this section we consider near-interval colorings. This edge coloring model was studied by Petrosyan et al. in [18] under the name interval $(t, 1)$-colorings. If $f$ is a proper edge coloring of a graph $G, v \in V(G)$, and $f(v)$ is near-interval, then we say that $f$ is near-interval at $v$.

In [18] Petrosyan et al. investigate the question of existence of near-interval colorings for different families of graphs; in particular, they give examples of bipartite graphs having no near-interval colorings. Here we are interested in the question whether $(a, b)$-biregular graphs have such colorings. This is a useful relaxation of Conjecture 1.1: for instance, for each positive integer $n \geq 4$, it is unknown whether all $(n-1, n)$-biregular graphs have interval colorings. However, it is an immediate consequence of König's edge coloring theorem that every such graph has a near-interval $n$-coloring. In fact, we have the following:

Proposition 3.1. If $G$ is a bipartite graph with $\delta(G)=n-1$ and $\Delta(G)=n$, then $G$ has a near-interval n-coloring.

The question whether Proposition 3.1 extends to general $(a, b)$-biregular graphs is open. In particular, it is unknown whether all $(n-2, n)$-biregular graphs have near-interval colorings if $n \geq 7$. The case $n=4$ has an affirmative solution by the result of [11]. We shall prove that all $(3,5)$ - and (4,6)-biregular graphs have near-interval colorings. For $(3,5)$-biregular graphs, this follows from Theorem 1.2.

Corollary 3.2. Every $(3,5)$-biregular graph has a near-interval 6-coloring.

Proof. Let $G=(X, Y ; E)$ be a $(3,5)$-biregular graph. Then $|X|=5 k$ and $|Y|=3 k$, for some positive integer $k$. Let $Y=\left\{y_{1}, \ldots, y_{3 k}\right\}$. From $G$ we form a $(3,6)$-biregular graph $H$ by

adding a set $X^{\prime}=\left\{x_{1}^{\prime}, \ldots, x_{k}^{\prime}\right\}$ of $k$ new vertices, and putting an edge between $x_{i}^{\prime}$ and each of the vertices $y_{3 i-2}, y_{3 i-1}, y_{3 i}$, for $i=1, \ldots, k$. The obtained graph $H$ is $(3,6)$-biregular, so by Theorem 1.2 it has an interval 7-coloring $f$. Moreover, it follows from the proof of Theorem 1.2 that if $x \in X$, then

$$
f(x) \in\{\{1,2,3\},\{2,3,4\},\{4,5,6\},\{5,6,7\}\} .
$$

Let $E_{1}=\{e \in E(G): f(e) \in\{3,5\}\}$, and note that $E_{1}$ covers $X$. Let $J$ be the subgraph of $G$ induced by the edges in $E_{1}$. Then each vertex of $X$ has degree 1 in $J$, and each vertex of $Y$ has degree 1 or 2 in $J$. Let $M$ be a (minimal) matching in $G-E_{1}$ saturating all vertices of $Y$ of degree 1 in $J$; such a matching exists by Hall's condition (or by König's edge coloring theorem). Next, let $J^{\prime}$ be the subgraph of $G$ induced by $E_{1} \cup M$. Since every vertex of $Y$ has degree 2 in $J^{\prime}$, the graph $G-E\left(J^{\prime}\right)$ has maximum degree 3. Define a proper 5-edge coloring $g$ of $G$ by properly coloring the edges of $G-E\left(J^{\prime}\right)$ with colors $1,2,5$, and the edges of $J^{\prime}$ with colors 3 and 4 . Since $E_{1}$ covers $X$, we have that $g(x) \neq\{1,2,5\}$ for each vertex $x \in X$. Moreover, $g(y)=\{1,2,3,4,5\}$ for each vertex $y \in Y$. This implies that $g$ is near-interval at every vertex $v$ of $G$ except if $v \in X$ and

$$
g(v) \in\{\{1,3,5\},\{1,4,5\}\} .
$$

From $g$ we define a new proper edge coloring $g^{\prime}$ of $G$ in the following way: for each edge $e=x y$, such that $x \in X, g(e)=1$ and $g(x) \in\{\{1,3,5\},\{1,4,5\}\}$, we set $g^{\prime}(e)=6$; we retain the color of every other edge of $G$. The obtained coloring $g^{\prime}$ is near-interval at every vertex of $G$. 
Note that a proper 5-edge coloring $f$ of a $(3,5)$-biregular graph $G=(X, Y ; E)$ is a nearinterval 5-coloring of $G$ if and only if $\{1,5\} \nsubseteq f(x)$ for each vertex $x \in X$. Moreover, since $G$ is $(3,5)$-biregular, $|X|=5 k$ and $|Y|=3 k$. Thus $|M(f, 1)|=|M(f, 5)|=3 k$, which implies that there is a vertex $x \in X$ such that $\{1,5\} \subseteq f(x)$. Hence, no (3,5)-biregular graph has a near-interval 5-coloring, which means that the number of colors used in Corollary 3.2 is best possible.

Let us now prove that all $(4,6)$-biregular graphs have near-interval 7-colorings.

Theorem 3.3. Every (4,6)-biregular graph has a near-interval 7-coloring.

Proof. Let $G=(X, Y ; E)$ be a $(4,6)$-biregular graph. Since all vertex degrees in $G$ are even, there is a closed eulerian trail $T$ in $G$. Let $E_{1}$ be the set of all even-indexed edges of $T$, and put $E_{2}=E(G) \backslash E_{1}$. Set $G_{1}=G\left[E_{1}\right]$ and $G_{2}=G\left[E_{2}\right]$, and note that $G_{i}$ is (2,3)-biregular for $i=1,2$. We define a proper edge coloring $f$ of $G$ using colors $2, \ldots, 7$ by properly coloring the edges of $G_{1}$ with colors $2,6,7$; and the edges of $G_{2}$ with colors $3,4,5$. Then $f(y)=\{2, \ldots, 7\}$ for each vertex $y \in Y$. Each vertex $x \in X$ receives two colors from $\{2,6,7\}$ and two colors from $\{3,4,5\}$ on its incident edges. This implies that if $f$ is not near-interval at some vertex $x \in X$, then

$$
f(x) \in\{\{2,3,4,7\},\{2,3,5,7\},\{2,4,5,7\}\} .
$$

For every such vertex $x$ we recolor the edge colored 7 incident to $x$ by color 1 . The obtained edge coloring is a near-interval 7-coloring of $G$.

Note that a proper 6-edge coloring $f$ of a $(4,6)$-biregular graph $G=(X, Y ; E)$ is a nearinterval 6-coloring of $G$ if and only if $\{1,6\} \nsubseteq f(x)$ for each vertex $x \in X$. Since $G$ is $(4,6)$-biregular, $|X|=3 k$ and $|Y|=2 k$, for some positive integer $k$. Thus, $|M(f, 1)|=2 k$ and $|M(f, 6)|=2 k$, which implies that $\{1,6\} \subseteq f(x)$ for some vertex $x \in X$. Hence, the number of colors used in Theorem 3.3 is best possible.

Next, we give two sufficient conditions for a $(4,6)$-biregular graph to admit an interval 8-coloring. Note that Hanson and Loten [12] proved that an interval coloring of an $(a, b)$ biregular graph uses at least $a+b-\operatorname{gcd}(a, b)$ colors, where gcd denotes the greatest common divisor. Hence, no (4,6)-biregular graph has an interval coloring with fewer than 8 colors.

Proposition 3.4. Let $G=(X, Y ; E)$ be a $(4,6)$-biregular graph. If there are partitions $X=X_{1} \cup X_{2} \cup X_{3}$ and $Y=Y_{1} \cup Y_{2}$, such that either

(i) $G\left[X_{i} \cup Y_{j}\right]$ is 2-regular for $i \in\{1,2,3\}$ and $j \in\{1,2\}$, or

(ii) $G\left[X_{2} \cup Y_{i}\right]$ is 2-regular for $i=1,2$, and $G\left[X_{1} \cup Y_{1}\right]$ and $G\left[X_{3} \cup Y_{2}\right]$ is 4-regular,

then $G$ has an interval 8-coloring.

Proof. Let $G=(X, Y ; E)$ be a $(4,6)$-biregular graph satisfying (i) or (ii) of Proposition 3.4. Suppose first that (i) holds. Then there are partitions $X=X_{1} \cup X_{2} \cup X_{3}$ and $Y=Y_{1} \cup Y_{2}$, such that $G\left[X_{i} \cup Y_{j}\right]$ is 2-regular for $i \in\{1,2,3\}$ and $j \in\{1,2\}$. By König's edge coloring theorem there is a proper edge coloring using 2 colors of each $G\left[X_{i} \cup Y_{j}\right]$. We define an edge coloring of $G$ by properly coloring the edge set of each $G\left[X_{i} \cup Y_{j}\right]$ using colors $2(i+j)-3,2(i+j)-2$, 
for $i=1,2,3$ and $j=1,2$. It is straightforward to verify that the obtained coloring is interval at every vertex of $G$.

Suppose now that (ii) holds. Then there are partitions $X=X_{1} \cup X_{2} \cup X_{3}$ and $Y=Y_{1} \cup Y_{2}$, such that $G\left[X_{2} \cup Y_{i}\right]$ is 2-regular for $i=1,2$, and $G\left[X_{1} \cup Y_{1}\right]$ and $G\left[X_{3} \cup Y_{2}\right]$ are both 4-regular. We properly color the edges of $G$ as follows (again using König's edge coloring theorem):

- color the edges of $G\left[X_{1} \cup Y_{1}\right]$ with colors $1,2,3,4$;

- color the edges of $G\left[X_{3} \cup Y_{2}\right]$ with colors $5,6,7,8$;

- color the edges of $G\left[X_{2} \cup Y_{i}\right]$ with colors $8-2 i-1,8-2 i$, for $i=1,2$.

It is straightforward to verify that the obtained coloring is interval at every vertex of $G$.

Since (4,6)-biregular graphs satisfying condition (i) or (ii) of Proposition 3.4 is the edgedisjoint union of regular bipartite graphs, Proposition 3.4 establishes existence of interval colorings for an infinite family of $(4,6)$-biregular graphs.

On the other hand, any (4,6)-biregular graph satisfying (i) or (ii) of Proposition 3.4 has a 4-regular subgraph covering all vertices of degree 6; and it is easy to construct examples of $(4,6)$-biregular graphs having no such subgraph. Consider, for instance, a $(4,6)$-biregular graph $G=(X, Y ; E)$ where $Y=\{1,2, \ldots, 8\}$ and the neighborhoods of the vertices in $X$ are twelve 4 -subsets $A_{1}, \ldots, A_{12}$ of $\{1,2, \ldots, 8\}$. $G$ has a 4-regular subgraph covering $Y$ if and only if there are four 4 -subsets among $A_{1}, \ldots, A_{12}$ such that each of the numbers $1, \ldots, 8$ belongs to exactly two of these 4-subsets. An explicit example failing this condition is

$$
\begin{gathered}
A_{1}=\{1,2,3,4\}, A_{2}=\{1,2,3,4\}, A_{3}=\{1,2,5,6\}, A_{4}=\{1,2,5,6\}, \\
A_{5}=\{1,2,7,8\}, A_{6}=\{1,2,7,8\}, A_{7}=\{3,5,7,8\}, A_{8}=\{3,5,7,8\}, \\
A_{9}=\{4,5,6,8\}, A_{10}=\{4,5,6,8\}, A_{11}=\{3,4,6,7\}, A_{12}=\{3,4,6,7\} .
\end{gathered}
$$

Straightforward case analysis shows that there are no four 4 -subsets such that each of the numbers $1, \ldots, 8$ belongs to exactly two of these 4 -subsets. Hence, the corresponding (4,6)-biregular graph has no 4-regular subgraph covering $Y$ and therefore does not satisfy the hypothesis of Proposition 3.4. However, it is an easy exercise to prove that this graph does have an interval coloring with 8 colors.

Note further that since the proof of Proposition 3.4 relies only on the application of König's edge coloring theorem, which is valid in the multigraph setting as well, the proof works equally well for (4,6)-biregular multigraphs. The latter also holds for Theorem 3.3.

\section{4 (4,8)-biregular graphs}

In this section we consider (4,8)-biregular graphs. We will prove that all $(4,8)$-biregular graphs have cyclic interval colorings, but let us first briefly discuss the question of the existence of interval 8-colorings of $(4,8)$-biregular graphs. Let $G=(X, Y ; E)$ be a $(4,8)$-biregular graph. If $f$ is an interval 8-coloring of $G$, then the edges with colors in $\{1,2,3,4\}$ span a 4-regular subgraph of $G$ covering $Y$; and the edges with colors in $\{5,6,7,8\}$ span a 4-regular graph covering $Y$. Conversely, if $G$ has two edge-disjoint 4-regular graphs covering $Y$, then it clearly has an interval 8-coloring. Thus we have the following: 
Proposition 4.1. A (4,8)-biregular graph has an interval 8-coloring if and only if it has a 4-regular subgraph covering the vertices of degree 8.

Unfortunately, the problem of determining whether a (4,8)-biregular graph has a 4-regular subgraph covering all vertices of degree 8 is $\mathcal{N} \mathcal{P}$-complete, as the following proposition states. The proof is deferred to the appendix.

Proposition 4.2. The problem of determining whether a $(4,8)$-biregular graph has a 4-regular subgraph covering all vertices of degree 8 is $\mathcal{N} \mathcal{P}$-complete.

Propositions 4.1 and 4.2 imply the following:

Corollary 4.3. The problem of determining whether a $(4,8)$-biregular graph has an interval 8-coloring is $\mathcal{N} \mathcal{P}$-complete.

We now turn our attention to cyclic interval colorings and first note the following analogue of Proposition 3.1 for cyclic interval colorings.

Proposition 4.4. If $G$ is a bipartite graph with $\delta(G)=n-1$ and $\Delta(G)=n$, then $G$ has a cyclic interval n-coloring.

It follows from Proposition 4.4 that all $(n-1, n)$-biregular graphs have cyclic interval colorings. Note further that any interval coloring of a bipartite graph $G$ can be transformed into a cyclic interval $\Delta(G)$-coloring by taking all colors modulo $\Delta(G)$. So Conjecture 1.1 in fact has the following weaker consequence for which the answer is unknown:

Conjecture 4.5. Every $(a, b)$-biregular graph has a cyclic interval $\max \{a, b\}$-coloring.

The smallest unsolved case of Conjecture 4.5 is $(a, b)=(3,5)$. In [6] we gave some conditions for a $(3,5)$-biregular graph to admit a cyclic interval coloring; such a graph $G=$ $(X, Y ; E)$ has a cyclic interval 5 -coloring if it has a 3 -regular subgraph covering $Y$, or a $(3,4)$-biregular subgraph covering $Y$. Here we prove that all $(4,8)$-biregular graphs have cyclic interval 8-colorings.

Theorem 4.6. Every (4,8)-biregular graph has a cyclic interval 8-coloring.

Proof. Let $G=(X, Y ; E)$ be a $(4,8)$-biregular graph. Since all vertex degrees in $G$ are even, $G$ has a closed Eulerian trail $T$ with an even number of edges. Let $E_{1}$ be the set of all even-indexed edges in $G$, and put $E_{2}=E(G) \backslash E_{1}$. Set $G_{1}=G\left[E_{1}\right]$ and $G_{2}=G\left[E_{2}\right]$, and note that $G_{i}$ is $(2,4)$-biregular for $i=1,2$.

By Theorem 2.1, each $G_{i}$ has an interval 4-coloring such that each vertex in $X$ receives colors 1,2 or 3,4 on its incident edges. Let $f_{i}$ be such a coloring of $G_{i}, i=1,2$. From $f_{1}$ we define a new proper edge coloring $g_{1}$, by replacing colors 3 and 4 by 5 and 6 , respectively; from $f_{2}$ we define a new proper edge coloring $g_{2}$ by replacing colors 1 and 2 by colors 7 and 8 , respectively. Note that if $y \in Y$, then $g_{1}(y) \cup g_{2}(y)=\{1, \ldots, 8\}$.

If $x \in X$, then $g_{1}(x) \in\{\{1,2\},\{5,6\}\}$ and $g_{2}(x) \in\{\{3,4\},\{7,8\}\}$. Hence, $g_{1}$ and $g_{2}$ together form a cyclic interval 8-coloring of $G$.

Finally, let us remark that the proof of Theorem 4.6 is also valid for multigraphs, since Theorem 2.1 holds for multigraphs. 


\section{References}

[1] A. S. Asratian, C. J. Casselgren, On interval edge colorings of $(\alpha, \beta)$-biregular bipartite graphs, Discrete Math. 307 (2007), 1951-1956.

[2] A. S. Asratian, C. J. Casselgren, Jennifer Vandenbussche, D. B. West, Proper path factors and interval edge-coloring of (3,4)-biregular bigraphs, Journal of Graph Theory 61 (2009), 88-97.

[3] A. S. Asratian, R. R. Kamalian, Investigation of interval edge-colorings of graphs, Journal of Combinatorial Theory. Series B 62 (1994), no. 1, 34-43.

[4] A. S. Asratian, R. R. Kamalian, Interval coloring of the edges of a multigraph (in Russian), Applied mathematics 5, Yerevan University, (1987), 25-34.

[5] M. A. Axenovich, On interval colorings of planar graphs, Congressus Numerantium 159 (2002), 77-94.

[6] C. J. Casselgren, B. Toft, On interval and cyclic interval edge colorings of $(3,5)$-biregular graphs, in preparation.

[7] C. J. Casselgren, Some results on interval edge colorings of bipartite graphs, Master's thesis, Linköping University, 2005.

[8] K. Giaro, The complexity of consecutive $\Delta$-coloring of bipartite graphs: 4 is easy, 5 is hard, Ars Combin. 47 (1997), 287-298.

[9] K. Giaro, M. Kubale, Compact scheduling of zero-one time operations in multi-stage systems, Discrete Applied Mathematics 145 (2004), 95-103

[10] K. Giaro, M. Kubale, Consecutive edge-colorings of complete and incomplete Cartesian products of graphs, Congressus Numerantium 128 (1997), 143-149.

[11] H. M. Hansen, Scheduling with minimum waiting periods (in Danish), Master Thesis, Odense University, Odense, Denmark, 1992.

[12] D. Hanson, C. O. M. Loten, A lower bound for interval colouring bi-regular bipartite graphs, Bulletin of the ICA 18 (1996), 69-74.

[13] D. Hanson, C.O.M Loten, B. Toft, On interval colourings of bi-regular bipartite graphs, Ars Combinatoria 50 (1998), 23-32.

[14] T. R. Jensen, B. Toft, Graph Coloring problems, Wiley Interscience, 1995.

[15] A. V. Kostochka, Unpublished manuscript, 1995

[16] M. Kubale, A. Nadolski, Chromatic scheduling in a cyclic open shop, European Journal of Operational Research 164, (2005), 585-591.

[17] A. Nadolski, Compact cyclic edge-colorings of graphs, Discrete Mathematics 308, (2008), 2407-2417. 
[18] P. A. Petrosyan, H. Z. Arakelyan, V. M. Baghdasaryan A generalization of interval edge-colorings of graphs, Discrete Applied Mathematics 158 (2010), 1827-1837.

[19] A. V. Pyatkin, Interval coloring of $(3,4)$-biregular bipartite graphs having large cubic subgraphs, Journal of Graph Theory 47 (2004), 122-128.

[20] S. V. Sevastjanov, Interval colorability of the edges of a bipartite graph (in Russian), Metody Diskretnogo Analiza, 50 (1990), 61-72.

[21] M. Stiebitz, B. Toft, D. Scheide, L.M. Favrholdt, Graph edge colouring: Vizing's theorem and Goldberg's conjecture, Wiley Interscience, 2012.

[22] Fan Yang, Xiangwen Li, Interval coloring of (3, 4)-biregular bigraphs having two $(2,3)$ biregular bipartite subgraphs, Applied Mathematics Letters 24 (2011), 1574-1577.

\section{Appendix}

In this section we give the proofs of Propositions 2.5 and 4.2 .

Proof of Proposition 2.5 (sketch). Let $G=(X, Y ; E)$ be a $(3,6)$-biregular graph. From $G$ we shall construct a $(3,9)$-biregular graph $H$ with parts $X_{H}$ and $Y_{H}$ such that $H$ has a 3-regular subgraph covering $Y_{H}$ if and only if $G$ has a 3-regular subgraph covering $Y$. By Proposition 2.4 , this suffices for proving the proposition.

Suppose that $Y=\left\{y_{1}, \ldots, y_{k}\right\}$. Let $K_{3,8}^{(1)}, \ldots, K_{3,8}^{(6)}$ be 6 pairwise disjoint copies of the complete bipartite graph $K_{3,8}$, and let $K_{4,2}^{(1)}, \ldots, K_{4,2}^{(3)}$ be 3 pairwise disjoint copies of the complete bipartite graph $K_{4,2}$. Set $\mathcal{K}=K_{3,8}^{(1)} \cup \cdots \cup K_{3,8}^{(6)}$ and $\mathcal{K}^{\prime}=K_{4,2}^{(1)} \cup K_{4,2}^{(1)} \cup K_{4,2}^{(3)}$. Suppose that the parts of $\mathcal{K}$ are

$$
T=\left\{t_{1}, \ldots, t_{48}\right\} \text { and } U=\left\{u_{1}, \ldots, u_{18}\right\},
$$

where the vertices in $U$ are labelled according to Figure 2. Suppose that the parts of $\mathcal{K}^{\prime}$ are

$$
A=\left\{a_{1}, \ldots, a_{12}\right\} \text { and } B=\left\{b_{1}, \ldots, b_{6}\right\},
$$

where the vertices of $A$ and $B$ are labelled according to Figure 2. Let $W=\left\{w_{1}, w_{2}, w_{3}\right\}$ and $Z=\left\{z_{1}, \ldots, z_{10}\right\}$ be two disjoint sets of new vertices distinct from all vertices in $G$ and not in any of the complete bipartite graphs. Let

$$
E_{w a}=\left\{w_{1} u_{1}, w_{1} u_{4}, w_{2} u_{7}, w_{2} u_{10}, w_{3} u_{13}, w_{3} u_{16}\right\},
$$

and for $i=1,2,3$, let

$$
E_{i}=\left\{a_{4 i-3} u_{6 i-4}, a_{4 i-2} u_{6 i-3}, a_{4 i-1} u_{6 i-1}, a_{4 i} u_{6 i}\right\} .
$$

We now define the auxiliary graph $J$ from $\mathcal{K} \cup \mathcal{K}^{\prime}$ and the vertices in $W$ by adding the new edges in $E_{w a}$ and $E_{1} \cup E_{2} \cup E_{3}$. From $J$ we define the graph $L$ by adding the vertices in $Z$ and letting the neighborhoods of these vertices be:

$$
N_{L}\left(z_{i}\right)=\left\{b_{1}, b_{2}, b_{3}\right\}, \text { for } i=1, \ldots, 5
$$


and

$$
N_{L}\left(z_{i}\right)=\left\{b_{4}, b_{5}, b_{6}\right\}, \text { for } i=6, \ldots, 10 .
$$

The graph $L$ is bipartite, all vertices in one part have degree 9 , and all vertices in the other part have degree 2 or 3 ; in fact $d_{L}(v)=2$ if and only if $v \in W$ (see Figure 2).

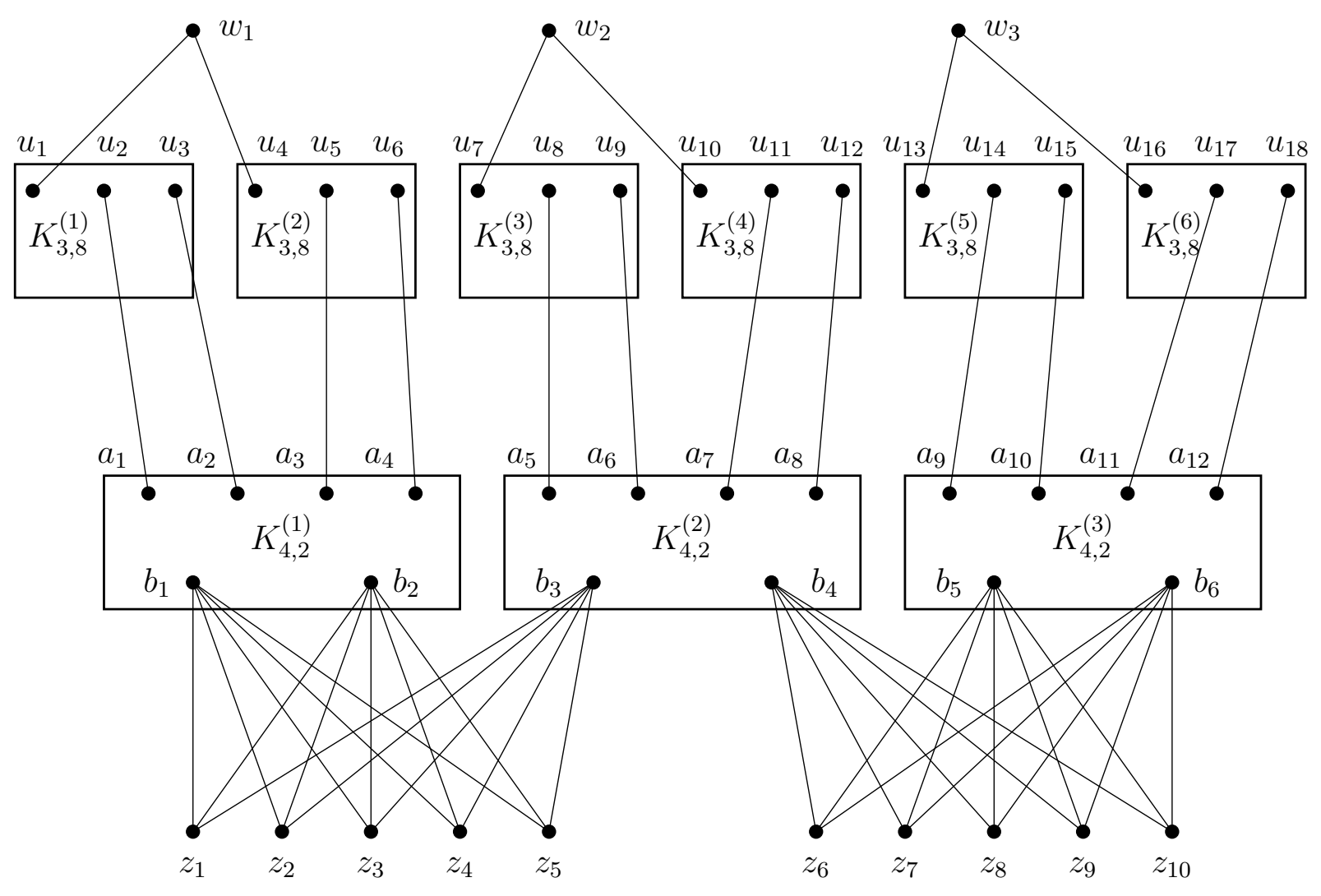

Figure 2: The graph $L$ in the proof of Proposition 2.5.

We take $k$ pairwise disjoint copies $L^{(1)}, \ldots, L^{(k)}$ of the graph $L$ and denote the vertices in each $L^{(i)}$ corresponding to

- $w_{1}, w_{2}, w_{3}$ by $w_{1}^{(i)}, w_{2}^{(i)}, w_{3}^{(i)}$,

- $a_{1}, \ldots, a_{12}$, by $a_{1}^{(i)}, \ldots, a_{12}^{(i)}$,

- $u_{1}, \ldots, u_{18}$ by $u_{1}^{(i)}, \ldots, u_{18}^{(i)}$, and

- $b_{1}, \ldots, b_{6}$ by $b_{1}^{(i)}, \ldots, b_{6}^{(i)}$,

respectively. Set $W_{i}=\left\{w_{1}^{(i)}, w_{2}^{(i)}, w_{3}^{(i)}\right\}$. Now we define the graph $H$ from $G$ and the copies of $L$ by for each $i=1, \ldots, k$ adding the edges in $\left\{y_{i} w_{1}^{(i)}, y_{i} w_{2}^{(i)}, y_{i} w_{3}^{(i)}\right\}$ to $G \cup L^{(1)} \cup \cdots \cup L^{(k)}$. The graph $H$ is $(3,9)$-biregular and we denote its parts by $X_{H}$ and $Y_{H}$, respectively. 
We will now prove that $G$ has a cubic subgraph covering $Y$ if and only if $H$ has a cubic subgraph covering $Y_{H}$. If $G$ has a cubic subgraph covering $Y$, then it is straightforward to verify from Figure 2 that $H$ has a cubic subgraph covering $Y_{H}$.

Conversely, suppose that $H$ has a cubic subgraph $Q$ covering $Y_{H}$. We will prove that none of the vertices in $W_{1} \cup \cdots \cup W_{k}$ are in $Q$. If this holds, then the part of $Q$ which is in $G$ is a 3-regular graph covering $Y$, which implies the desired result. Suppose, for a contradiction, that there are integers $i, j$ such that $w_{j}^{(i)}$ is in $Q$. Suppose for example that $j=1$. Since each of the vertices in $\left\{u_{1}^{(i)}, u_{2}^{(i)}, \ldots, u_{6}^{(i)}\right\}$ have degree 3 in $Q$, this implies that $\left\{a_{1}^{(i)}, a_{2}^{(i)}, a_{3}^{(i)}, a_{4}^{(i)}\right\} \subseteq V(Q)$. However, this implies that $d_{Q}\left(b_{1}^{(i)}\right) \geq 4$, a contradiction. The cases when $j \in\{2,3\}$ can be dealt with similarly. Thus we conclude that the part of $Q$ that lies in $G$ is 3-regular, as required.

Proof of Proposition 4.2 (Sketch). Let $G=(X, Y ; E)$ be a $(3,6)$-biregular graph. Consider the graph $J$ in Figure 3. Let $F$ be a fixed subgraph of $J$ induced by $s, w_{1}, w_{2}, w_{3}, a_{1}, a_{3}, a_{5}, b$, and all vertices of degree 6 in each copy of $K_{6,3}$, and three arbitrary vertices of degree three in each copy of $K_{6,3}$.

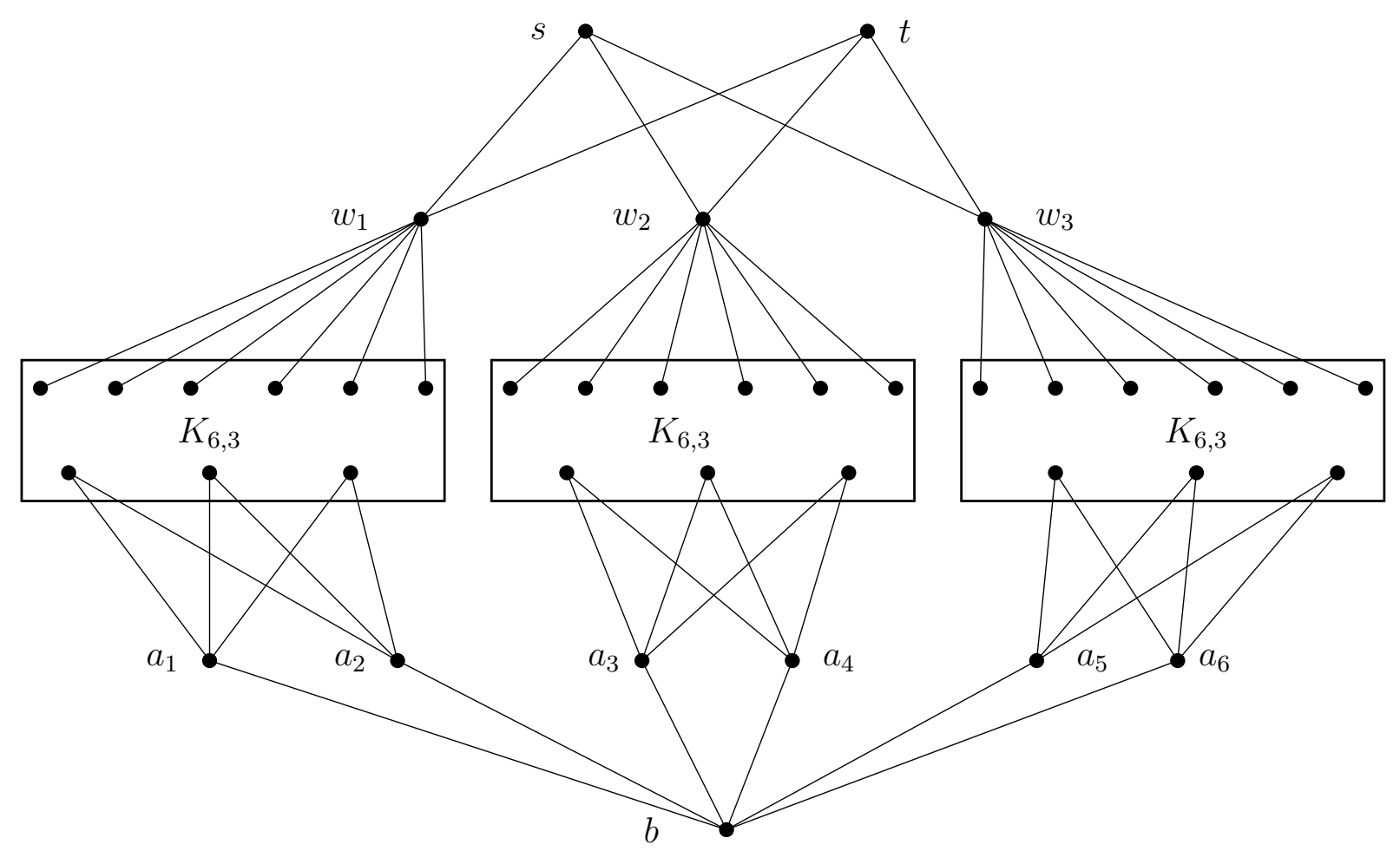

Figure 3: The graph $J$ in the proof of Proposition 4.2.

We shall construct a $(4,8)$-biregular graph $H$ from several copies of $G$ and $J$, such that $H$ has a 4-regular subgraph covering all vertices of degree 8 if and only if $G$ has a 3-regular subgraph covering $Y$. By Proposition 2.4, this will imply the desired result. 
Since $G$ is (3,6)-biregular, we have that $|X|=2 k$ and $|Y|=k$, for some positive integer $k$. Let

$$
X=\left\{x_{1}, \ldots, x_{2 k}\right\} \text { and } Y=\left\{y_{1}, \ldots, y_{k}\right\} .
$$

We take 4 pairwise disjoint copies $G^{(1)}, \ldots, G^{(4)}$ of the graph $G$, where each $G^{(j)}$ has parts

$$
X^{(j)}=\left\{x_{1}^{(j)}, \ldots, x_{2 k}^{(j)}\right\} \text { and } Y^{(j)}=\left\{y_{1}^{(j)}, \ldots, y_{k}^{(j)}\right\} .
$$

For each $i \in\{1, \ldots, k\}$ and each $j \in\{1,2,3,4\}$, let $J_{i}^{(j)}$ be a copy of the graph $J$; that is, $J_{1}^{(1)}, \ldots, J_{k}^{(4)}$ are $4 k$ pairwise disjoint copies of the graph $J$ in Figure 3 . For each $i \in\{1, \ldots, k\}$ and $j \in\{1,2,3,4\}$, let $F_{i}^{(j)}$ be the subgraph of $J_{i}^{(j)}$, corresponding to the subgraph $F$ of $J$, and set $\hat{F}=\cup_{i, j} F_{i}^{(j)}$. For each $J_{i}^{(j)}$, denote by $s_{i}^{(j)}, t_{i}^{(j)}, b_{i}^{(j)}$, the vertices corresponding to $s, t$ and $b$ in $J$, respectively (see Figure 3 ). We now form the auxiliary graph $L$ from $G^{(1)}, \ldots, G^{(4)}$ and $J_{1}^{(1)}, \ldots, J_{k}^{(4)}$ and a set of $2 k$ new vertices $Z=\left\{z_{1}^{(1)}, \ldots z_{k}^{(1)}, z_{1}^{(2)}, \ldots, z_{k}^{(2)}\right\}$ in the following way: for each $i \in\{1, \ldots, k\}$ and each $j \in\{1,2,3,4\}$,

- join $y_{i}^{(j)}$ and $s_{i}^{(j)}$ by an edge,

- join $y_{i}^{(j)}$ and $t_{i}^{(j)}$ by an edge, and

- join $b_{i}^{(j)}$ to $z_{i}^{(1)}$ and $z_{i}^{(2)}$ by two edges.

A schematic of the graph $L$ in the case when $k=3$ appears in Figure 4.

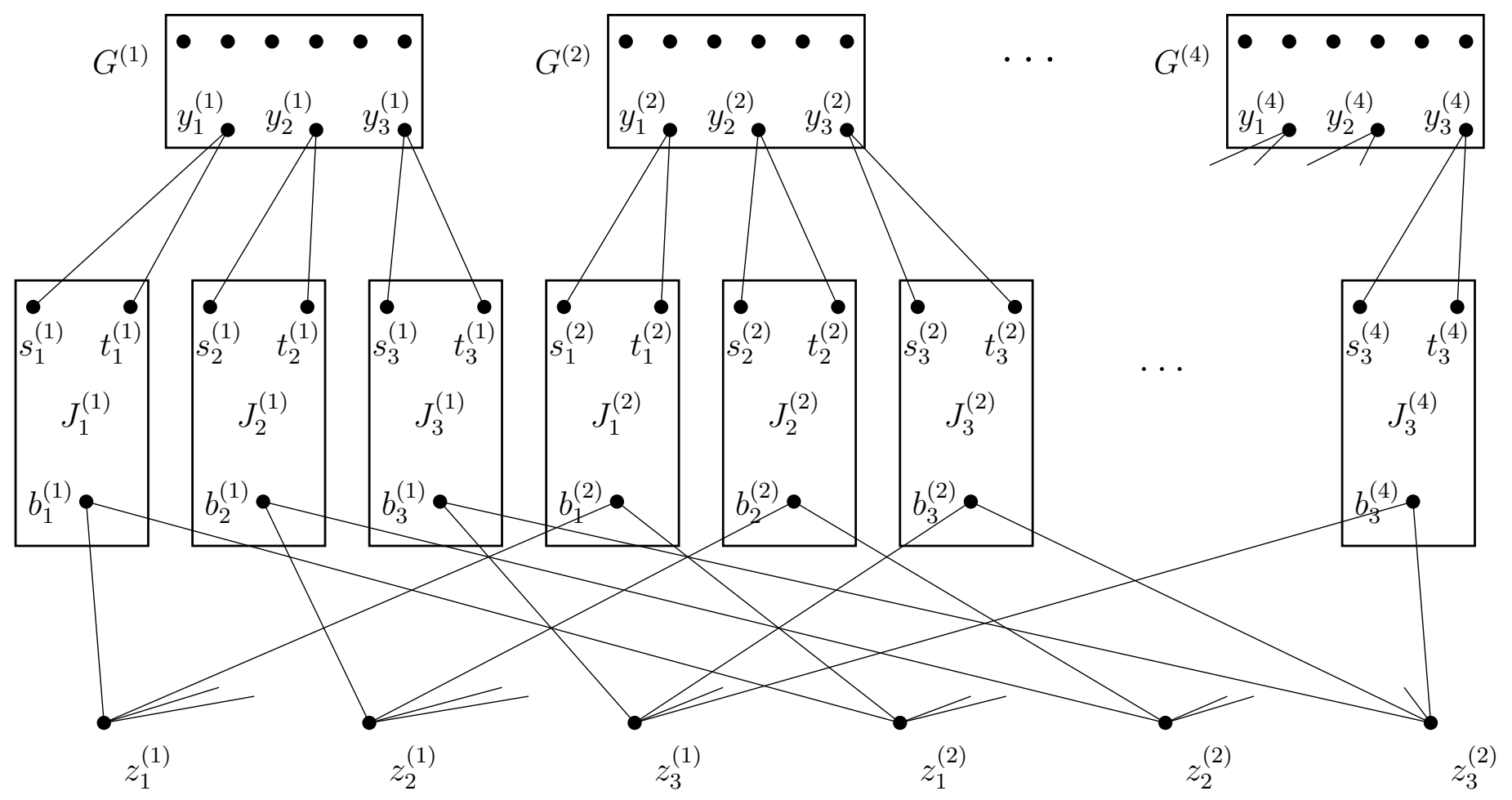

Figure 4: The graph $L$ in the case when $k=3$ in the proof of Proposition 4.2. 
Next, let $K_{4,7}^{(1)}, \ldots, K_{4,7}^{(2 k)}$ be $2 k$ pairwise disjoint copies of the complete bipartite graph $K_{4,7}$; and for each $K_{4,7}^{(i)}$, suppose that the parts of $K_{4,7}^{(i)}$ are

$$
C_{i}=\left\{c_{1}^{(i)}, \ldots, c_{4}^{(i)}\right\} \text { and } D_{i}=\left\{d_{1}^{(i)}, \ldots, d_{7}^{(i)}\right\},
$$

where vertices in $C_{i}$ all have degree 7 , and vertices in $D_{i}$ all have degree 4 . We are now ready to form the graph $H$ from $L$ and $K_{4,7}^{(1)}, \ldots, K_{4,7}^{(2 k)}$ by for each $i=1, \ldots, 2 k$ and $j=1,2,3,4$, joining $x_{i}^{(j)}$ with $c_{j}^{(i)}$ by an edge. The obtained graph $H$ is $(4,8)$-biregular. Set $C=\cup_{i=1}^{2 k} C_{i}$, and $D=\cup_{i=1}^{2 k} D_{i}$.

Suppose first that $G$ has a 3 -regular subgraph $Q$ that covers $Y$. Then for each $j \in$ $\{1,2,3,4\}, Q$ induces a 3 -regular subgraph $Q^{(j)}$ in $G^{(j)}$ covering $Y^{(j)}$. We set $\hat{Q}=\cup_{j} Q^{(j)}$. Let $I \subseteq\{1, \ldots, 2 k\}$ be a set defined by $i \in I$ if and only if $x_{i}$ is in $Q$. Let $I^{\prime}=\{1, \ldots, 2 k\} \backslash I$. We define the graph $R$ by letting it be the subgraph of $H$ induced by the vertices in

$$
\bigcup_{i \in I}\left\{d_{1}^{(i)}, d_{2}^{(i)}, d_{3}^{(i)}\right\} \cup \bigcup_{i \in I^{\prime}}\left\{d_{1}^{(i)}, d_{2}^{(i)}, d_{3}^{(i)}, d_{4}^{(i)}\right\} \cup C \cup\left\{z_{1}^{(1)}, \ldots, z_{k}^{(1)}\right\} \cup V(\hat{Q} \cup \hat{F})
$$

The graph $R$ is 4-regular and covers all vertices of degree 8 in $H$.

Now suppose that $H$ has a 4-regular subgraph $S$ covering all vertices of degree 8 . We shall prove that $S$ induces a 3-regular subgraph of $G^{(1)}$ that covers $Y^{(1)}$. If this holds, then clearly $G$ has a 3 -regular subgraph covering $Y$.

Each vertex of $X^{(1)} \cap V(S)$ has degree 3 in the subgraph of $H$ induced by $V\left(G^{(1)}\right) \cap V(S)$. Hence, we only need to prove that each vertex of $Y^{(1)}$ has degree 3 in the subgraph of $H$ induced by $V\left(G^{(1)}\right) \cap V(S)$. Let $y_{i}^{(1)} \in Y^{(1)}$. Suppose first that none of $s_{i}^{(1)}$ and $t_{i}^{(1)}$ are in $S$. It is straightforward to verify from Figure 3 that then $b_{i}^{(1)}$ cannot be adjacent to any vertex of $J_{i}^{(1)}$ in $S$ and thus $d_{S}\left(b_{i}^{(1)}\right) \leq 2$, which contradicts that $S$ is 4-regular and covers all vertices of degree 8 in $H$.

Now suppose that both $s_{i}^{(1)}$ and $t_{i}^{(1)}$ are in $S$. Again, from Figure 3 one may verify that this implies that $d_{S}\left(b_{i}^{(1)}\right) \geq 6$, a contradiction as well. Thus we conclude that exactly one of the vertices in $\left\{s_{i}^{(1)}, t_{i}^{(1)}\right\}$ is in $S$. Hence, $y_{i}^{(1)}$ has degree 3 in the subgraph of $H$ induced by $V\left(G^{(1)}\right) \cap V(S)$, as required. 